\title{
Sedation versus general anesthesia for transcatheter aortic valve replacement
}

\author{
Keita Sato ${ }^{1}$, Philip M. Jones ${ }^{1,2}$ \\ ${ }^{1}$ Department of Anesthesia \& Perioperative Medicine, ${ }^{2}$ Department of Epidemiology \& Biostatistics, University of Western Ontario, London, ON, \\ Canada \\ Contributions: (I) Conception and design: PM Jones; (II) Administrative support: PM Jones; (III) Provision of study materials or patients: All authors; \\ (IV) Collection and assembly of data: K Sato; (V) Data analysis and interpretation: All authors; (VI) Manuscript writing: All authors; (VII) Final \\ approval of manuscript: All authors. \\ Correspondence to: Philip M. Jones, MD, MSc. Room C3-110, University Hospital, London Health Sciences Centre, 339 Windermere Rd, London, \\ ON N6A 5A5, Canada. Email: philip.jones@lhsc.on.ca.
}

\begin{abstract}
There is currently significant controversy regarding the best anesthesia management for patients undergoing transcatheter aortic valve replacement (TAVR). Some institutions primarily use general anesthesia (GA) but many institutions primarily use moderate sedation. Much of the controversy is due to the limited evidence base available to inform this decision and the strong feelings and pre-conceived notions about the optimal anesthesia technique which exist amongst anesthesiologists, cardiologists, and cardiac surgeons. In this article and in the context of TAVR, we will define the salient differences between GA and sedation, review the results and limitations of the currently available data, and discuss the priority questions for future research.
\end{abstract}

Keywords: Sedation; local anesthesia; general anesthesia (GA); transcatheter aortic valve replacement (TAVR); transcatheter aortic valve implantation

Submitted Jul 04, 2018. Accepted for publication Aug 16, 2018.

doi: $10.21037 /$ jtd.2018.08.89

View this article at: http://dx.doi.org/10.21037/jtd.2018.08.89

\section{What is the difference between sedation and general anesthesia (GA)?}

A variety of terminology has been used to refer to sedation for medical procedures. This includes conscious sedation, local anesthesia, monitored anesthesia care (MAC), and procedural sedation and analgesia (1). Now that the expression of 'conscious sedation' is considered as oxymoron since the association of two terms, conscious and sedation, is contradictory (2), procedural sedation/ analgesia is the preferred term. Sedation and analgesia comprises a continuum of states ranging from minimal sedation through GA $(1,3)$. Procedural moderate sedation and analgesia is defined as a drug-induced depression of consciousness during which patients respond purposefully to verbal commands, either alone or accompanied by light stimulation (3). No interventions are needed to maintain a patent airway if spontaneous breathing is adequate, unlike GA. Cardiovascular function is usually maintained. Hereafter, sedation indicates procedural moderate sedation and analgesia unless otherwise noted.

Sedation is a minimal mode of anesthesia, in which intubation is not required, which can potentially reduce respiratory complications in elderly and frail patients [as is often the case in transcatheter aortic valve replacement (TAVR) candidates]. On the other hand, transesophageal echocardiography (TEE) is often impractical with sedated patients during TAVR. This may conceivably have a negative impact on outcomes in TAVR since immediate and potentially life-threatening complications [such as a significant paravalvular leak (PVL), annulus rupture, and cardiac tamponade] may fail to be diagnosed when TEE is not used. In addition, unexpected movements of patients under sedation at an inopportune time may be a concern 
and potentially catastrophic, particularly during valve deployment.

Potential advantages of using sedation instead of GA for TAVR are a shortened procedure time due to avoidance of intubation and extubation (4-9), less requirements of vasopressors during the procedure (7), decreased respiratory complications (9), reduced intensive care unit (ICU) and hospital lengths of stay $(5,6,9,10)$, and reduced costs $(8)$, On the contrary, using TEE during TAVR under sedation may not be as sensitive as TEE and could possibly lead to an increased incidence of PVL in patients under sedation $(8,11)$. Also, conversion from sedation to GA may contribute to increased mortality. These factors need to be taken into consideration when discussing short- and long-term morbidity and mortality in TAVR under sedation versus GA.

\section{Literature comparing sedation with GA in TAVR}

At the time of writing, there is but one randomized clinical trial comparing sedation versus GA. This RCT investigated cumulative cerebral desaturation using near-infrared spectroscopy during the procedure, and its outcomes were neurological and respiratory complications in patients undergoing TAVR, not "harder" clinical outcomes such as procedural time, hospital length of stay, or procedural success (12). Several national or European registry-based observational studies with or without propensity score analysis $(4,6,9,10,13-15)$, a few meta-analyses $(11,16,17)$, and small observational studies (15-25) have also been reported regarding early or intermediate outcomes (Table 1).

\section{Influence of anesthetic technique on short-term outcomes}

Most studies have reported similarly high success rate of the procedure under sedation or GA, ranging around from $95 \%$ to $99 \%$, with the majority of studies around $97 \%(9,10,14,16)$. One study (10) found that sedation was associated with lower procedural success $(98 \%$ vs. $99 \%$, $\mathrm{P}<0.001$ ), which is clearly not clinically significant (and would need to verified in other studies even if true).

Although most studies (4-8,11,13-17) demonstrated similar in-hospital and 30-day mortality rates and major adverse event rates, two large (16,543 patients and 10,997 patients) observational studies $(9,10)$ reported a significant reduction of mortality and major adverse complications including stroke when sedation was used compared to GA. Husser et al. (9) reported a lower 30-day mortality with sedation versus GA (crude sample: $3.5 \%$ vs. $4.9 \%, \mathrm{P}<0.001$; after adjustment using matching: $2.8 \%$ vs. $4.6 \%, \mathrm{P}<0.001$ ) in a German registry study of 16,543 patients undergoing TAVR, in which $49 \%$ of patients received sedation. However, all of the procedural complications like device malposition, embolization, conversion to sternotomy, and vascular complications were significantly more frequent in patients under GA. This suggests a probable chronological or selection bias in the effect of sedation versus GA, since when TAVR is first being adopted and clinicians are at an early phase of the learning curve of TAVR, GA is usually chosen. Hyman et al. (10) demonstrated that sedation was associated with a lower in-hospital mortality incidence $(1.5 \%$ vs. $2.4 \%, \mathrm{P}<0.001)$ after inverse probability of exposureweighted adjustment, in the STS/ACC transcatheter valve registry of TAVR. In their study, 1,737 patients (16\%) had sedation for the procedure.

In some studies, reduced ICU length of stay has been reported with sedation compared to GA in patients undergoing TAVR (7-10), while in other studies, no differences in ICU stay was demonstrated $(4,14,15)$. The duration of ICU stays of patients with either sedation or GA is significantly different among the studies. Mean ICU stays in patents with sedation or GA in these studies varies from 0 to 48 hours, and from 15.5 hours to 3.5 days, respectively. This may simply reflect a significant variety of institutional protocols for TAVR in the real world, but it ably demonstrates the significant heterogeneity that exists in this literature. Likewise, there are several studies which reported reduced hospital stay with sedation compared to GA $(5,6,10,11,16)$, whereas no difference was found in other studies $(4,14)$, Mean hospital stay with sedation is reported as from 2 to 9 days, and with GA, from 4 to 9 days. Cost-effectiveness analysis of sedation versus GA in TAVR has seldom been performed. Babaliaros et al. (8) found that a 'minimalist approach' of TAVR in the catheter laboratory under sedation was associated with a reduced ICU length of stay [minimalist approach 22 (interquartile range, IQR: $2-28 \mathrm{~h}$ ), $\mathrm{n}=70 \mathrm{vs}$. standard approach 28 (IQR: 23-48 h), n=72; $\mathrm{P}<0.001]$ and a reduced cost [minimalist approach $\$ 45,485$ (standard deviation, SD: $\$ 14,397), \mathrm{n}=70$ vs. standard approach \$55,377 (SD: \$22,587), $\mathrm{n}=72 ; \mathrm{P}<0.001]$ compared to TAVR performed in a hybrid operating room under GA in an observational study of 142 patients.

Increased PVL after TAVR in patients with sedation has been reported in a meta-analysis (11). On the other hand, others have found no differences in PVL in patients under sedation versus GA $(8,9,14,15)$. Oguri et al. (14) showed 


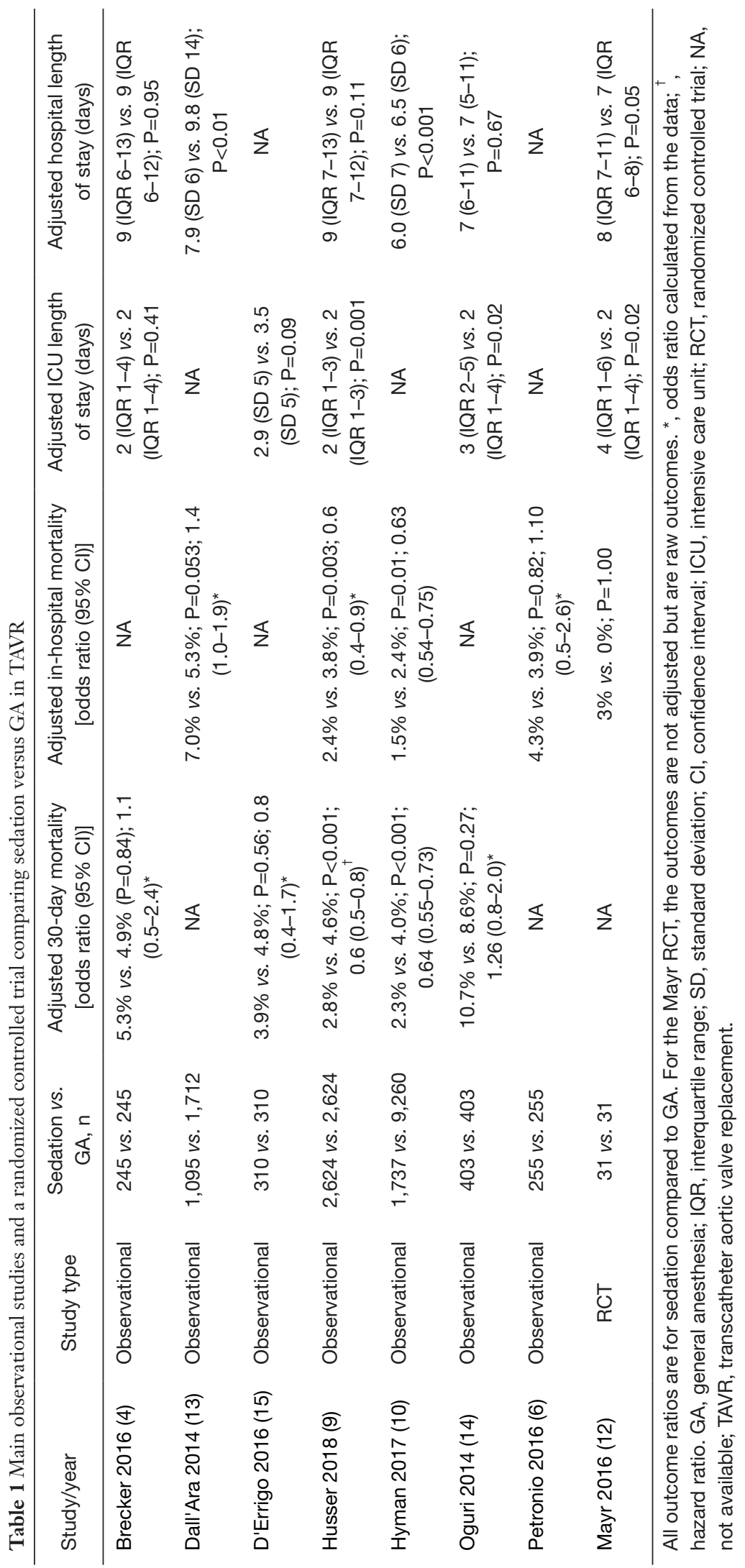


increased PVL of no less than mild with sedation in the crude sample $(19 \%$ vs. $15 \%, \mathrm{P}=0.015)$ but this effect was no longer present after controlling for confounding using propensity score-matching $(16 \% v s .13 \%, \mathrm{P}=0.19)$ in the French registry of 2,326 patients. Making interpretation more difficult is the fact that the definition of PVL or criteria for picking up patients with PVL are significantly different among the studies. For example, no less than mild PVL was used by Oguri et al., but more than mild was used by Husser et al. $(9,14)$.

Red blood cell transfusion incidence in TAVR has been investigated in several small observational studies and one large observational study using a European registry $(13,18-24)$. In one meta-analysis of these studies, a lower rate of red blood cell transfusion was reported (risk ratio 0.69, $95 \%$ CI: 0.49 to 0.96 ) (17). Catecholamine treatment during the procedure was similarly examined in small studies $(19,20,22,23,25)$, and found to be lower in patients with sedation than GA in a meta-analysis (risk ratio 0.47 , 95\% CI: 0.32 to 0.70$)$ (17). In all the studies where the procedural time was studied, a shorter procedural time has been constantly reported in patients with sedation $(4-9,11,16)$. In the largest observational study, the procedural time of TAVR under sedation was significantly shorter than GA (67 vs. $77 \mathrm{~min}, \mathrm{P}<0.001)$, although the clinical significance of this finding is unclear (9).

The conversion rate from sedation to GA for any reason is reported to be from $5 \%$ to $6 \%$ in a small number of large observational studies and meta-analyses $(4,10,16,17)$. In almost all the studies, the conversion rate was not reported and thus it is unclear how converted cases were dealt with in the analysis in the studies. In a prospective RCT in 62 patients under sedation versus GA, Mayr et al. found that adverse events, including bradypnea and the need for airway maneuvers, were more frequent in patients undergoing TAVR under sedation. However, their primary outcome of cerebral desaturation was not different between the groups (12). Furthermore, their conversion rate of $19 \%$ from sedation to GA was extremely high, which could indicate that their protocol of sedation might not have been adequate.

Overall, observational studies have demonstrated that sedation is associated with similar short-term morality but with potentially shorter ICU and hospital stays compared to GA. Sedation may be associated with increased PVL, a decreased need for vasopressor support, and reduced blood transfusions.

\section{Influence of anesthetic technique on intermediate- and long-term outcomes}

There are four large observational studies in which intermediate-term outcomes of 1- or 2-year mortality was studied $(4,8,9,13,14)$. All of the studies reported no differences in intermediate-term mortality between sedation and GA. Husser et al. (9) reported no difference of 1 -year mortality (sedation $14 \%$ vs. GA $16 \%, \mathrm{P}=0.13$ ) in a propensity score-matched population of 5,248 patients. Brecker et al. also found no difference of 2-year mortality and stroke rate (2-year adjusted mortality: sedation $25 \% v s$. GA $24 \%, \mathrm{P}=0.78$; 2 -year stroke rate: $5 \%$ vs. $7 \%, \mathrm{P}=0.57$ ) in a propensity score-matched analysis of 490 patients (4).

\section{Limitations of the studies and future direction of the research}

The fact that there are significant methodological limitations in all of the research investigating anesthetic techniques for TAVR is the most important message of this review. First, selection bias due to the lack of clear criteria for choosing sedation or GA likely exists in all of the studies. Patients undergoing TAVR are often frail with significant comorbidities. Thus, GA can often be arbitrarily selected for patients with an increased frailty or difficult procedural anatomy. These factors may plausibly affect many adverse outcomes if they are imbalanced between the sedation and GA groups in clinical studies. For example, vascular complications and bleeding during TAVR were more common in patients with GA in the matched sample in the largest observation study (9). Although methods for controlling confounding, such as propensity score matching, will adjust for known and measured confounders, every observational study will have a problem with residual confounding (and the inability to control for unknown or unmeasured prognostic factors influencing outcomes).

Second, chronological bias must be taken into consideration. GA is usually chosen for TAVR at the first stage of learning the procedure because it is safer when unexpected complications happen and it permits long procedural times without patient discomfort. In the study using French Registry study, sedation for TAVR increased from 14\% in January 2010 to $59 \%$ in October 2011 in the studied period (14). This means that more experienced institutions use sedation instead of GA, which may create the apparition that sedation itself leads to better outcomes. Indeed, in the previously described German registry, the 
most experienced centers used sedation in $64 \%$ of the cases, while the least experienced institutions used sedation in only $38 \%$ of the cases (9). Furthermore, the changes of TAVR devices can affect this temporal bias of the studies of sedation versus GA. Newer-generation TAVR devices are emerging with smaller delivery systems and improved designs to reduce PVL, and they have been reported to have better outcomes (26). Again, if the newer-generation devices are used more under sedation, there may appear to be better outcomes with sedation. Unfortunately, the generation of the TAVR devices have rarely been described or controlled for in the studies, whereas the decreased use of GA over years has been constantly shown $(6,9,10,14)$.

Third, the conversion rate from sedation to GA was rarely recorded in the large-scale registries, and it is unclear how the converted cases were dealt with in most of the studies. Conversion can occur when encountering difficult vascular access, bleeding, or significant procedural complications. Consequently, patients converted to GA can have an increased ICU or hospital length of stay. As mentioned before, since conversion rate was reported not to be rare, around $6 \%$, these cases should be correctly documented and dealt with based on an intention-to-treat analysis, not necessarily censored at the time of conversion.

Fourth, the preoperative planning of TAVR, especially sizing of the transcatheter valve, should be the same between sedation versus GA. Currently, computed tomography (CT) measurements of the aortic valve anulus are considered to be the gold standard (27). However, originally TEE was used for the valve sizing. Since TEE measurement of the annulus has proven to be smaller than CT on average (28), if the CT was not used for selecting the size of the valve, it may lead to a higher incidence of PVL as well as a lower incidence of complete AV block and new pacemaker implantation. In a meta-analysis, a lower incidence of pacemaker implantation was found in patients undergoing TAVR under GA (17). How could GA itself prevent postoperative pacemaker implantation in TAVR? This finding may be direct evidence of significant residual confounding in these small observational studies.

Fifth, the choice of drugs used for sedation and depth of sedation has rarely been described in the observational studies. This is a limitation with many retrospective observational studies. Since the choice of drugs for sedation in TAVR can affect the respiratory state and the need for vasopressor support (29), at least the primary drugs (and dosages) used for sedation should be noted in studies comparing sedation with GA.
Finally, the protocols of fast-track pathways for both sedation and GA patients should be fairly and equally established. If a hospital has an institutional protocol that mandates that patients with GA need to stay a night in ICU just because they were tracheally intubated, and that patients with sedation may bypass the ICU because they were not intubated, it will necessarily demonstrate a prolonged ICU and hospital length of stay in patients undergoing TAVR under GA. Unfortunately, because of the retrospective nature of the observational studies, not all of the studies are clear regarding their exact protocol of postoperative management.

Sedation has been increasingly used in TAVR over time in Europe (ranging from $50 \%$ to $75 \%$ in the literature) and in North America $(6,10,13,14)$. The increase is especially apparent in high volume centers (9). This trend seems destined to continue since the current best evidence suggests that sedation is not inferior to GA, and sedation is associated with shorter ICU and hospital length of stay. However, the best available evidence is almost exclusively derived from observational studies with the significant limitations mentioned above. Currently, no consensus exists regarding the selection criteria for sedation in TAVR and each institution has their own protocols. High-risk patients due to cardiac compromise or anatomical reasons including vascular access, predicted difficult airway, severe pulmonary hypertension, or CPAP therapy for obstructive sleep apnea may potentially be excluded from the candidates for sedation in TAVR (12). Given the expected increase in the number of TAVR procedures being performed due to expanded indications and device funding, further studies, the only way to truly know if there is an important difference between sedation and GA for TAVR is to perform high quality RCTs. Due to the limitations of the currently available evidence, it is still worthwhile to conduct RCTs addressing this question, so that we ensure we are providing the best quality care to our patients. Ultimately, the goal is to identify the patients who are likely to benefit from the differential selection of sedation or GA in TAVR.

\section{Acknowledgements}

None.

\section{Footnote}

Conflicts of Interest: The authors have no conflicts of interest to declare. 


\section{References}

1. Hinkelbein J, Lamperti M, Akeson J, et al. European Society of Anaesthesiology and European Board of Anaesthesiology guidelines for procedural sedation and analgesia in adults. Eur J Anaesthesiol 2018;35:6-24.

2. Green SM, Krauss B. Procedural sedation terminology: moving beyond “conscious sedation". Ann Emerg Med 2002;39:433-5.

3. Practice Guidelines for Moderate Procedural Sedation and Analgesia 2018: A Report by the American Society of Anesthesiologists Task Force on Moderate Procedural Sedation and Analgesia, the American Association of Oral and Maxillofacial Surgeons, American College of Radiology, American Dental Association, American Society of Dentist Anesthesiologists, and Society of Interventional Radiology. Anesthesiology 2018;128:437-79.

4. Brecker SJ, Bleiziffer S, Bosmans J, et al. Impact of Anesthesia Type on Outcomes of Transcatheter Aortic Valve Implantation (from the Multicenter ADVANCE Study). Am J Cardiol 2016;117:1332-8.

5. Jabbar A, Khurana A, Mohammed A, et al. Local Versus General Anesthesia in Transcatheter Aortic Valve Replacement. Am J Cardiol 2016;118:1712-6.

6. Petronio AS, Giannini C, De Carlo M, et al. Anaesthetic management of transcatheter aortic valve implantation: results from the Italian CoreValve registry. EuroIntervention 2016;12:381-8.

7. Pani S, Cagino J, Feustel P, et al. Patient Selection and Outcomes of Transfemoral Transcatheter Aortic Valve Replacement Performed with Monitored Anesthesia Care Versus General Anesthesia. J Cardiothorac Vasc Anesth 2017;31:2049-54.

8. Babaliaros V, Devireddy C, Lerakis S, et al. Comparison of transfemoral transcatheter aortic valve replacement performed in the catheterization laboratory (minimalist approach) versus hybrid operating room (standard approach): outcomes and cost analysis. JACC Cardiovasc Interv 2014;7:898-904.

9. Husser O, Fujita B, Hengstenberg C, et al. Conscious Sedation Versus General Anesthesia in Transcatheter Aortic Valve Replacement: The German Aortic Valve Registry. JACC Cardiovasc Interv 2018;11:567-78.

10. Hyman MC, Vemulapalli S, Szeto WY, et al. Conscious Sedation Versus General Anesthesia for Transcatheter Aortic Valve Replacement: Insights from the National Cardiovascular Data Registry Society of Thoracic Surgeons/American College of Cardiology Transcatheter
Valve Therapy Registry. Circulation 2017;136:2132-40.

11. Maas EH, Pieters BM, Van de Velde M, et al. General or Local Anesthesia for TAVI? A Systematic Review of the Literature and Meta-Analysis. Curr Pharm Des 2016;22:1868-78.

12. Mayr NP, Hapfelmeier A, Martin K, et al. Comparison of sedation and general anaesthesia for transcatheter aortic valve implantation on cerebral oxygen saturation and neurocognitive outcomedagger. Br J Anaesth 2016;116:90-9.

13. Dall'Ara G, Eltchaninoff H, Moat N, et al. Local and general anaesthesia do not influence outcome of transfemoral aortic valve implantation. Int J Cardiol 2014;177:448-54.

14. Oguri A, Yamamoto M, Mouillet G, et al. Clinical outcomes and safety of transfemoral aortic valve implantation under general versus local anesthesia: subanalysis of the French Aortic National CoreValve and Edwards 2 registry. Circ Cardiovasc Interv 2014;7:602-10.

15. D'Errigo P, Ranucci M, Covello RD, et al. Outcome After General Anesthesia Versus Monitored Anesthesia Care in Transfemoral Transcatheter Aortic Valve Replacement. J Cardiothorac Vasc Anesth 2016;30:1238-43.

16. Frohlich GM, Lansky AJ, Webb J, et al. Local versus general anesthesia for transcatheter aortic valve implantation (TAVR)--systematic review and metaanalysis. BMC Med 2014;12:41.

17. Ehret C, Rossaint R, Foldenauer AC, et al. Is local anaesthesia a favourable approach for transcatheter aortic valve implantation? A systematic review and meta-analysis comparing local and general anaesthesia. BMJ Open 2017;7:e016321.

18. Bergmann L, Kahlert P, Eggebrecht H, et al. Transfemoral aortic valve implantation under sedation and monitored anaesthetic care--a feasibility study. Anaesthesia 2011;66:977-82.

19. Covello RD, Ruggeri L, Landoni G, et al. Transcatheter implantation of an aortic valve: anesthesiological management. Minerva Anestesiol 2010;76:100-8.

20. Dehedin B, Guinot PG, Ibrahim H, et al. Anesthesia and perioperative management of patients who undergo transfemoral transcatheter aortic valve implantation: an observational study of general versus local/regional anesthesia in 125 consecutive patients. J Cardiothorac Vasc Anesth 2011;25:1036-43.

21. Gauthier C, Astarci P, Baele P, et al. Mid-term survival after transcatheter aortic valve implantation: Results with respect to the anesthetic management and to the access 
route (transfemoral versus transapical). Ann Card Anaesth 2015;18:343-51.

22. Goren O, Finkelstein A, Gluch A, et al. Sedation or general anesthesia for patients undergoing transcatheter aortic valve implantation--does it affect outcome? An observational single-center study. J Clin Anesth 2015;27:385-90.

23. Motloch LJ, Rottlaender D, Reda S, et al. Local versus general anesthesia for transfemoral aortic valve implantation. Clin Res Cardiol 2012;101:45-53.

24. Yamamoto M, Meguro K, Mouillet G, et al. Effect of local anesthetic management with conscious sedation in patients undergoing transcatheter aortic valve implantation. Am J Cardiol 2013;111:94-9.

25. Balanika M, Smyrli A, Samanidis G, et al. Anesthetic management of patients undergoing transcatheter aortic valve implantation. J Cardiothorac Vasc Anesth 2014;28:285-9.

Cite this article as: Sato K, Jones PM. Sedation versus general anesthesia for transcatheter aortic valve replacement. J Thorac Dis 2018;10(Suppl 30):S3588-S3594. doi: 10.21037/jtd.2018.08.89
26. Barbanti M, Buccheri S, Rodes-Cabau J, et al. Transcatheter aortic valve replacement with newgeneration devices: A systematic review and meta-analysis. Int J Cardiol 2017;245:83-9.

27. Holmes DR Jr, Mack MJ, Kaul S, et al. 2012 ACCF/ AATS/SCAI/STS expert consensus document on transcatheter aortic valve replacement. J Am Coll Cardiol 2012;59:1200-54.

28. Jilaihawi H, Kashif M, Fontana G, et al. Cross-sectional computed tomographic assessment improves accuracy of aortic annular sizing for transcatheter aortic valve replacement and reduces the incidence of paravalvular aortic regurgitation. J Am Coll Cardiol 2012;59:1275-86.

29. Mayr NP, Wiesner G, van der Starre P, et al. Dexmedetomidine versus propofol-opioid for sedation in transcatheter aortic valve implantation patients: a retrospective analysis of periprocedural gas exchange and hemodynamic support. Can J Anaesth 2018;65:647-57. 